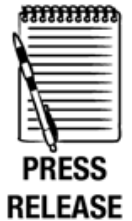

- Additional appendix is published online only. To view this file please visit the journal online (http://jme.bmj.com).

${ }^{1}$ King's College London, London, UK

${ }^{2}$ Queen Mary, University of London, London, UK

\section{Correspondence to \\ Dr Marianne Promberger, King's College London, Health \\ Psychology, Fifth Floor Bermondsey Wing, Guy's Hospital, London SE1 9RT, UK marianne.promberger@kcl.ac.}

Received 28 July 2010 Revised 22 March 2011 Accepted 14 April 2011 Published Online First 13 June 2011

\title{
Acceptability of financial incentives to improve health outcomes in UK and US samples
}

\author{
Marianne Promberger, ${ }^{1}$ Rebecca C H Brown, ${ }^{2}$ Richard E Ashcroft, ${ }^{2}$ \\ Theresa M Marteau ${ }^{1}$
}

\section{ABSTRACT}

In an online study conducted separately in the UK and the US, participants rated the acceptability and fairness of four interventions: two types of financial incentives (rewards and penalties) and two types of medical interventions (pills and injections). These were stated to be equally effective in improving outcomes in five contexts: (a) weight loss and (b) smoking cessation programmes, and adherence in treatment programmes for (c) drug addiction, (d) serious mental illness and (e) physiotherapy after surgery. Financial incentives (weekly rewards and penalties) were judged less acceptable and to be less fair than medical interventions (weekly pill or injection) across all five contexts. Context moderated the relative preference between rewards and penalties: participants from both countries favoured rewards over penalties in weight loss and treatment for serious mental illness. Only among US participants was this relative preference moderated by perceived responsibility of the target group. Overall, participants supported funding more strongly for interventions when they judged members of the target group to be less responsible for their condition, and vice versa. These results reveal a striking similarity in negative attitudes towards the use of financial incentives, rewards as well as penalties, in improving outcomes across a range of contexts, in the UK and the USA. The basis for such negative attitudes awaits further study.

\section{INTRODUCTION}

Financial incentives are increasingly being used to improve outcomes by motivating health-related behaviour change. These include incentive schemes aimed at enhancing outcomes of existing treatments such as drug abstinence programmes and taking medication to prevent relapse of medical conditions such as psychosis and stroke. ${ }^{1-3}$ Incentives are also being used to achieve sustained weight loss and smoking cessation. ${ }^{4-6}$ While the evidence of effectiveness is limited and mixed in many contexts, there is good evidence for their effectiveness in drug abstinence programmes and to a lesser extent, for smoking cessation in pregnancy. ${ }^{17}$

All the schemes described above involve the use of rewards. While negative incentives are implicit in taxes on tobacco and alcohol and in higher insurance premiums tied to behavioural risk factors such as smoking, explicit negative incentives are less often used than reward schemes and have been evaluated less frequently. Negative incentive schemes are currently mostly seen in the form of self-binding contracts involving individuals pledging their own money or betting with others on success or failure to attain health-related targets, ${ }^{8}$ although evidence on the effectiveness of such contracts is absent.

Even if effective, offering financial incentives to motivate health-enhancing behaviour attracts opprobrium in lay and professional media, being criticised for unfairly rewarding bad behaviour and being coercive. ${ }^{9} 10$ We report here on a systematic attempt to describe and understand the acceptability of different types of financial incentives across a range of health contexts. We set out to compare the acceptability of financial incentives with similarly effective medical interventions, as well as the use of penalties compared with rewards. We presented financial incentives as interventions additional to existing programmes, rather than as rewards for changing health behaviours per se. We also explored the extent to which perceived responsibility for needing healthcare moderates the acceptability of rewards compared with penalties. We conducted the study separately with participants from the UK and the US, the two highincome countries where financial incentive schemes are most often being used. We had no hypotheses about specific differences between these countries. Given the stark differences between their healthcare systems, which also made it necessary to slightly adapt the wording, we analysed the data for each participant group separately.

We predicted that participants would show less support for equally effective health-related interventions involving financial incentives than medically-based interventions. We also predicted that rewards would be favoured over penalties for some recipient groups of healthcare, and penalties over rewards for others. More specifically, we predicted that penalties would be favoured over rewards when recipients were deemed responsible for their condition, and vice versa.

\section{METHOD}

The study used a within-subjects design: 2 (type of intervention: financial vs medical) $\times 2$ (valence of intervention: positive vs negative) $\times 5$ (basic treatment scenario: smoking cessation, mental illness, drug addiction, weight loss, physical therapy).

\section{Participants}

The study was conducted online using a UK subject panel (http://participate-in-research.org.uk) and a US subject panel (http://www.sas.upenn.edu/ baron/ q.html). Country of residence was identified as follows: the US panel is run by Jonathan Baron, who has address data for the members of his panel and 
who sent out a notification email for the study only to members holding US addresses. Members of the UK Participate in Research panel submit information about their country of residence when they sign up on the website, and again, notification email was sent only to such members who had reported the UK as country of residence.

The US panel has been established over about 10 years and consists of 1200 participants who closely match the 2000 US census in demographic characteristics with the exception of being slightly better educated on average and with an overrepresentation of women. ${ }^{11}$ The UK panel of the study is newly established and, at the time of the study, consisted of about 150 participants, about equal numbers of men and women; further demographic data are not yet available. Participants of both panels found them online, partly through links from other websites. For the UK panel, participants had also been actively recruited through online ads offering pay of about $£ 2.00-3.00$ for participation in online academic research.

We removed two UK participants from the data who had completed the questionnaire extremely quickly and whose answers indicated that they did not take it seriously.

The included UK participants comprised 45 women and 43 men, aged between 18 and 81 years $(M=48, S D=14.31)$. They were paid $£ 3.00$ for completion of the questionnaire.

US participants comprised 70 women and 30 men, aged between 23 and 67 years $(M=44.43, S D=11.00)$. They were paid US\$3.50 for completion of the questionnaire.

\section{Procedure and materials}

On the first page, participants saw an introduction message. UK participants read that they would see hypothetical scenarios about medical treatments offered by the UK NHS along with interventions the NHS was considering funding in addition to these treatments. They were told to assume that the proposed interventions all 'cost the same amount of money, per person, for the same increase in effectiveness of the basic treatment'. Participants were first told: 'Imagine your country has a national health insurance programme. This programme is funded through taxes. It pays for many medical treatments for all residents, but not all treatments are covered'. Subsequently, all references to the NHS were replaced by 'the national health insurance'

On the following pages, participants saw at the top of each page a one-sentence summary of the existing treatment, followed by a two-sentence description of the proposed add-on intervention.

Basic (existing) treatments comprised: a smoking cessation programme, outpatient services for patients with mental illness, treatment to reduce misuse of heroin and cocaine, weight loss treatment, physical therapy following knee replacement surgery.

Add-on interventions comprised: a pill, an injection, an incentive scheme involving penalties and an incentive scheme paying rewards.

Details of the materials used are provided in the online appendix.

For each participant, the order of these $5 \times 4=20$ treatment $\times$ intervention combinations was randomised and presented on separate pages, each followed by the same questions assessing acceptability and perceptions of fairness. Figure 1 gives an example page. This was followed by three more pages with a question about perceived pleasantness of the interventions, perceived responsibility for the underlying medical problem and questions about demographic characteristics.
The NHS currently funds treatment to help overweight people lose weight.

An Incentive scheme exists to make the treatment more effective.

The incentive scheme requires overweight people to pay a penalty each week they fail to lose a predefined amount of weight.

Do you think this add-on intervention should be funded by the NHS?

definitely yes $O \quad 0 \quad 0 \quad 0 \quad 0$ definitely not

How fair do you think it would be to implement this add-on intervention?

very fair $\mathrm{O} \circ \mathrm{O} \bigcirc \mathrm{O}$ very unfair

Figure 1 Example page for treatment $\times$ intervention combinations and questions.

\section{Measures}

Each of the 20 pages with a treatment $\times$ add-on intervention combination was followed by a question about acceptability and a question about perceptions of fairness:

\section{Acceptability}

Do you think this add-on intervention should be funded by the NHS?

Definitely yes-definitely not (7-point scale, ranging from +3 to -3$)$.

\section{Fairness}

How fair do you think it would be to implement this add-on intervention?

Very fair-very unfair (7-point scale, ranging from +3 to -3 ).

Following the 20 treatment $\times$ intervention combinations, we included a manipulation check for perceived pleasantness or unpleasantness of the interventions.

\section{Perceived pleasantness of the interventions}

How pleasant or unpleasant do you think it is (eg, to be given an injection every week)?

Very unpleasant-very pleasant (7-point scale, ranging from -3 to +3$)$.

On the next page, each participant rated the perceived responsibility for the condition underlying the basic treatment.

\section{Perceived responsibility}

How responsible do you think (smokers) are for (smoking)?

They are entirely responsible-they are not at all responsible (7-point scale, ranging from +3 to -3 ).

On the last two pages, we asked participants about relevant characteristics of their own health behaviour or medical history (eg, whether they were or had been overweight), and about their age and gender. Participants could choose 'prefer not to answer' for sensitive medical questions.

\section{Analysis}

Data were analysed using the statistical package $\mathrm{R}^{12}$ To test for differences in acceptability between medical versus financial incentive interventions, we collapsed across interventions involving pills and injections to form medical interventions, collapsed across interventions involving rewards and penalties to form financial interventions, and then conducted t tests testing for a difference between these groups, by collapsing answers across basic treatments to the mean per subject and conducting paired $t$ tests of those means. To test for differences in relative acceptability of rewards versus penalties between target groups, 
we looked only at answers involving financial incentives and compared the relative preference for penalties versus rewards across different target groups by conducting an analysis of variance (ANOVA) with basic treatment and type of incentive as the independent factors, with acceptability as the dependent variable, and tested for an interaction. To test for influence of perceived responsibility, we conducted mixed models using the $\mathrm{R}$ package lme4, specifying for each model crossed random effects for subjects and basic treatment scenarios, an analysis that takes account of differences between subjects and scenarios by modelling them. ${ }^{13}$ To test for the influence of perceived responsibility on overall funding support, we entered perceived responsibility into the model as a fixed effect along type of intervention. To test for the influence of perceived responsibility on differential support of rewards versus penalties, we included type of incentive (reward vs penalty), perceived responsibility and their interaction as fixed effects (the interaction would show the effect). We report CIs for the fixed effects based on Markov Chain Monte Carlo sampling $(n=10000)$ from the posterior distribution of the parameter estimates.

\section{RESULTS}

\section{Participants' experiences with the medical problems}

In all, $22 \%$ of UK participants and $19 \%$ of US participants were current smokers; 33\% (UK) and 49\% (US) currently overweight; $18 \%$ (both countries) had experience with mental health problems; $26 \%$ (UK) and 29\% (US) reported having taken recreational drugs other than alcohol; 24\% (UK) and 27\% (US) had had an operation requiring physical therapy. Participants' personal experience with the underlying problems had no effect on the results we report below.

\section{Perceived pleasantness of the interventions}

We had included a pill and an injection to mimic the hedonic valence of rewards compared with penalties. As predicted, a weekly injection was judged to be less pleasant (UK: $M=-1.23, S D=1.33$; US: $M=-1.10, S D=1.50)$ than a weekly pill (UK: $\mathrm{M}=0.69, \mathrm{SD}=1.49 ; \mathrm{t}(87)=-11.07 ; \mathrm{p}<0.001 ; \mathrm{US}$ : $\mathrm{M}=0.72, \mathrm{SD}=1.44 ; \mathrm{t}(99)=-10.41 ; \mathrm{p}<0.001)$, and a penalty was judged as less pleasant (UK: $M=-1.82, S D=1.31$; US: $M=-2.07$, $\mathrm{SD}=1.43$ ) than a reward (UK: $M=2.18, \mathrm{SD}=1.25 ; \mathrm{t}(87)=-17.59$; $\mathrm{p}<0.001$; US: $\mathrm{M}=2.06, \mathrm{SD}=1.47 ; \mathrm{t}(99)=-17.76$; $\mathrm{p}<0.001)$. We also predicted that financial incentives would evoke a starker hedonic judgement than medical interventions. For both panels, the difference in hedonic valence was stronger for financial incentives than for medical treatments (UK: $t(87)=8.02$; $\mathrm{p}<0.001$; US: $\mathrm{t}(99)=9.7 ; \mathrm{p}<0.001)$.

\section{Perceived responsibility of those requiring treatment}

As predicted, some target groups were perceived to be more responsible for their conditions than were others, see table 1 Among UK and US participants, there was strong agreement that smokers are responsible for smoking, that patients with mental health issues are not responsible for their illness, and that drug addicts are responsible for their addiction. Judgements were less pronounced and less unanimous for patients who are overweight and those requiring knee surgery.

Table 1 Perceived responsibility (mean (SD)) for problem

\begin{tabular}{llllll}
\hline & $\begin{array}{l}\text { Drug } \\
\text { addiction }\end{array}$ & $\begin{array}{l}\text { Mental health } \\
\text { problems }\end{array}$ & \multicolumn{3}{l}{$\begin{array}{l}\text { Knee } \\
\text { Overweight }\end{array}$} \\
\hline replacement & Smoking \\
\hline UK $(\mathrm{n}=88)$ & $1.78(1.45)$ & $-2.35(1.02)$ & $1.39(1.37)$ & $-1.16(1.93)$ & $2.01(1.45)$ \\
US $(\mathrm{n}=100)$ & $1.93(1.29)$ & $-1.97(1.22)$ & $1.15(1.22)$ & $-0.34(1.72)$ & $2.13(1.26)$ \\
\hline
\end{tabular}

\section{Acceptability of financial incentives}

Financial incentive interventions were perceived as less acceptable than medical interventions by UK and US participants (figure 2).

For the UK participants, the mean answer, across subjects and basic treatments, to the acceptability question was -0.05 (mean $\mathrm{SD}$ for scenarios $=2.30$ ) for the financial incentive interventions and 1.31 (mean SD of scenarios $=1.79$ ) for the medical interventions. This difference is significant $(\mathrm{t}(87)=-7.62, \mathrm{p}<0.001$; 95\% CI of the mean of differences: -1.72 to -1.01$)$.

For the US participants, the mean was -0.13 (mean SD for scenarios $=2.32$ ) for the financial incentive interventions and 1.26 (mean SD for scenarios=1.81) for the medical interventions, this, too, a significant difference $(\mathrm{t}(99)=-9.41, \mathrm{p}<0.001 ; 95 \% \mathrm{CI}$ of the mean of differences: -1.68 to -1.09$)$.

\section{Perceived fairness of financial incentives}

Financial incentives were perceived by UK and US participants as less fair than medical interventions.

For the UK participants, the mean answer to the question about fairness was -0.05 (mean SD for scenarios $=2.28$ ) for the financial incentive interventions and 1.20 (mean SD for scenarios $=1.82$ ) for the medical interventions, a significant difference $(\mathrm{t}(87)=-7.42, \mathrm{p}<0.001 ; 95 \% \mathrm{CI}$ of the mean of differences: -1.58 to -0.91$)$.

For the US participants, the mean answer to the question about fairness was -0.23 (mean SD for scenarios $=2.22$ ) for the financial incentive interventions and 1.11 (mean SD for scenarios $=1.78$ ) for the medical interventions, also a significant difference $(t(99)=-9.08, p<0.001 ; 95 \%$ CI of the mean of differences: -1.64 to -1.05$)$.

For UK and US panels, we calculated the correlation of each participants' answers to these two questions; the mean withinparticipant correlation was 0.75 for $U K$ participants $(S D=0.32)$ and 0.82 for US participants $(S D=0.22)$ : participants tend to think that an intervention should be funded when they think implementing it is fair, and vice versa.

\section{Rewards versus penalties}

Rewards were judged more acceptable than penalties in some but not all contexts (figure 3). In a within-subject ANOVA (dependent variable: funding support; independent variables: type of intervention, basic treatment), the interaction between type of intervention (rewards vs penalties) and basic treatment was significant. For the UK participants, $F(4)=4.07, p=0.003$; for the US participants, $\mathrm{F}(4)=4.81, \mathrm{p}<0.001$.

Participants from both countries favoured rewards over penalties in the contexts of helping patients who are overweight lose weight and of outpatient services for patients with mental health issues. The predicted effect of perceived responsibility on acceptability of rewards versus penalties was found only in US, but not UK participants: in a mixed model, the interaction between type of financial incentive (rewards vs penalties) and perceived responsibility is significant for the US panel $(\beta=-0.16$, $\mathrm{SE}=0.05, \mathrm{t}=-3.14,95 \% \mathrm{CI}=-0.27$ to -0.06$)$, but not for the UK panel ( $\beta=-0.07, \mathrm{SE}=0.05, \mathrm{t}=-1.35,95 \% \mathrm{CI}=-0.19$ to 0.03 ).

\section{Direct influence of perceived responsibility on acceptability}

Figure 1 shows that across all intervention types (medical and financial), there was more support for funding of interventions for some treatment groups than for others. For UK participants but not those from the US, funding was more strongly supported when the target group was not perceived responsible for their condition. The fixed effect of perceived responsibility 
Figure 2 Acceptability of funding (means) of different interventions in five contexts.
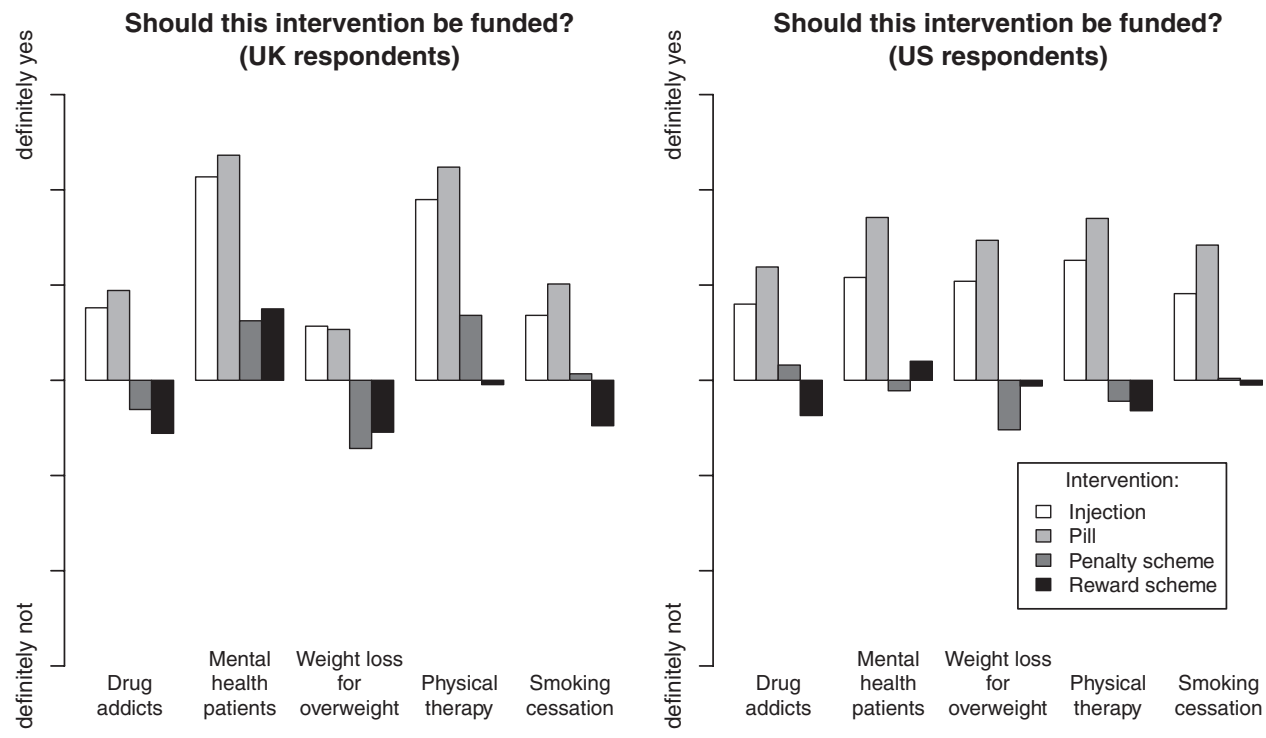

for UK participants was $-0.20 \quad(\mathrm{SE}=0.03, \mathrm{t}=-6.53,95 \%$ $\mathrm{CI}=-0.27$ to -0.13$)$; for US participants it was -0.05 ( $\mathrm{SE}=0.02$, $\mathrm{t}=-2.51,95 \% \mathrm{CI}=-0.09$ to 0 ).

\section{DISCUSSION}

Given equal effectiveness, financial incentives, whether rewards or penalties, are judged as less acceptable than medical interventions. We found this across five different health contexts and in participants from the UK and US. There are two possible sets of explanations for these findings. The first set assumes that these are valid results: participants based their judgements on the stated assumption that the financial and medical interventions were equally effective, and perceive incentives as less acceptable. This raises two questions: first, what explains these attitudes? Second, would incentives be seen as more acceptable if they were more effective, or would people refuse to make such trade offs? There are several possible reasons why people may find financial incentives less acceptable. It may stem from a violation of a cultural norm, namely the use of money in the relationship between a doctor or healthcare provider and a patient. Much has been written about areas of life in which money is an acceptable form of exchange and those in which it is not, these boundaries varying across time and between cultures. $^{14-17}$ The perceived unacceptability of financial incentives in the current context may also stem from a marked sense of injustice about offering money, a fungible good, to those who, through their own behaviour, might have avoided this. Concerning the nature of the incentive, it is possible that incentives that are less exchangeable (eg, vouchers or vouchers prespecified only for exchange of goods related to improved health) may be more acceptable.

We found differential support for penalties over rewards depending on the context of the basic treatments. We found only limited evidence that this related to perceptions of responsibility. It is plausible that our question about responsibility tapped different constructs for the different medical conditions. For example, notions of responsibility for being addicted to drugs are plausibly tied more closely to moral judgement and attributions of blame than are notions of responsibility for a knee injury. Evidence exists that some health behaviours, such as cigarette smoking, can become 'moralised', ${ }^{18} 19$ potentially linking responsibility with personal blameworthiness.

The second set of explanations for these findings is that they are not valid that is, they do not represent people's true views. This may be because the manipulations did not work as intended. For example, although we had instructed participants to assume that all interventions were equally effective in improving outcomes, it is possible that participants did not
Figure 3 Differential support of penalties versus rewards for the different target groups (identified by basic treatment). Mean answers to the question 'Should this be funded?' $(-3$ : definitely not; 3 : definitely yes).
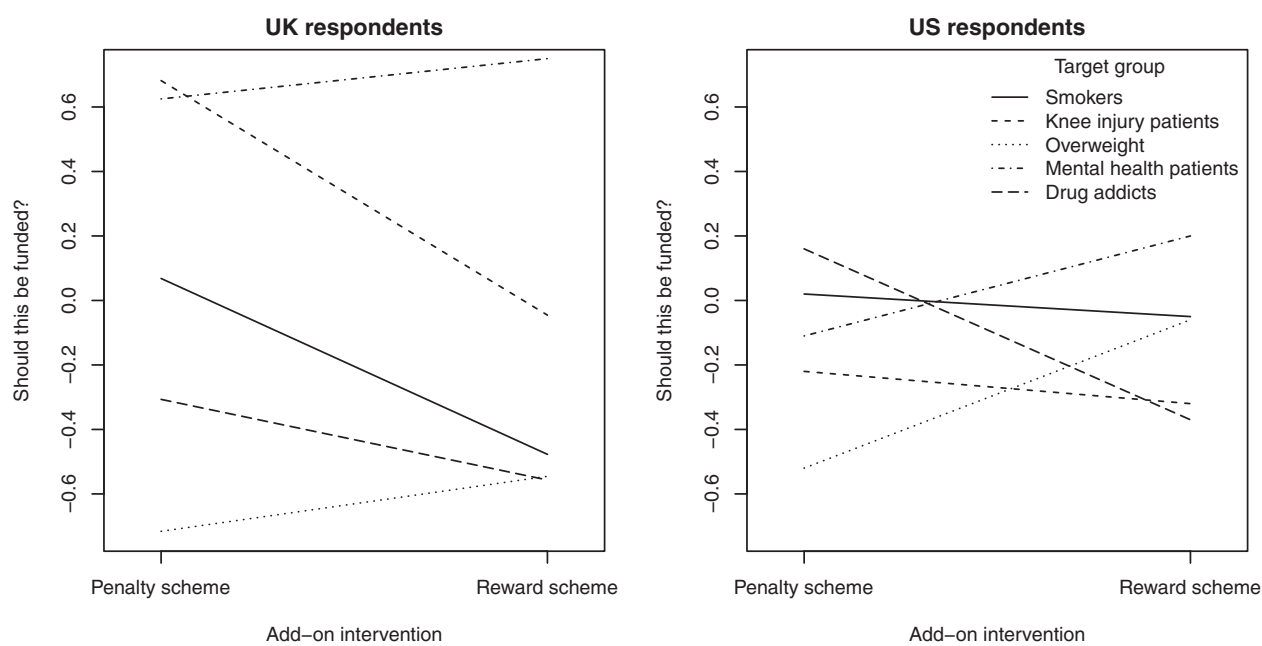
accept this assumption, resulting in perceived differences in effectiveness explaining differences in perceived acceptability. Respondents may simply believe that different interventions were differing in effectiveness, based on their own 'prior probabilities,' even if told that this was not so.

Replication and extension of the current study is needed to allow a more robust examination of the acceptability of financial incentives, using named as well as unnamed conditions, experimentally varied to assess the extent to which the acceptability of incentives is influenced by cost, effectiveness, perceived responsibility as well as type of reward (cash or vouchers for general or prespecified use).

Rejection of incentive schemes by the general public could all too easily be seen by politicians as a reason not to introduce them. This conclusion does not follow from our findings. To answer the question as to whether or not incentive schemes should be introduced would require more evidence on their consequences, intended and unintended, as well as in-depth analysis of the associated ethical issues of using incentives in healthcare. Perceptions about the acceptability of incentives, especially if accompanied by strong feelings, can be seen as a consequence, ${ }^{20}$ but the basis for such judgements are not fully understood. It may be the case that the results reported here stem from prejudice and illegitimate value judgements, and should thus not be reflected in policy decisions. For example, it is not clear how stable the preference for medical over financial interventions really is 'taboos' of using money in some contexts vary across cultures and may well change over time. ${ }^{14}$ As noted above, rejection of incentives may be based on beliefs about effectiveness or about unintended consequences, beliefs that can be challenged by evidence. Acceptance of different schemes may be subject to framing and presentation, or to personal experience, all of which vary and can be influenced. Even if a residual disutility for incentives in health contexts stubbornly persists, benefits may be great enough to justify overriding such concerns. At the other extreme, opposition to 'rewarding bad behaviour' might translate into the very tangible consequence of corroding the goodwill, and eroding the good behaviour, of those not rewarded for their virtue.

We note that 'acceptability' is a rather problematic concept. What we have not presented here is a survey of attitudes to incentives, which would inherently be time and context bound, sensitive to changes in salient background, such as exposure to recent news reports of incentive schemes or direct participation in such a scheme. Nevertheless, 'acceptability' is in some sense a relational property linking the incentive, the health behaviour, the respondent's attitudes and beliefs, and the wider context. And, as noted, acceptability is not inherently a normative concept that can be mapped directly onto right and wrong, fair or unfair. Nevertheless, the findings here presented do give some clues as to how experimentally informed evaluations of the fairness and moral rightness or wrongness of incentive schemes could be developed further, and of how the supposedly timeless intuitions of philosophers about these matters may be improved by the use of experimental studies.

\section{Strengths and limitations of the current study}

The present study is the first, to our knowledge, to examine the acceptability of using financial incentives, penalties and rewards, across a range of health contexts. While surveys have been conducted on the acceptability of using financial incentives in single contexts including compliance with drug treatment for psychosis and using rewards for smoking cessation, obesity, blood pressure control and diabetes management, ${ }^{3} 21$ this is the first study to our knowledge to assess acceptability of rewards and penalties across health contexts and in two countries. The scenarios we used can be seen as less controversial than some settings for incentives aimed at recipients of healthcare that are currently used or considered, such as paying for testing, paying for immunisation, or paying for adherence to a medication regimen with possible side effects. Our main finding of low acceptability of financial incentives could reasonably be expected to be even stronger for such scenarios. However, there may be a qualitative difference in the opposition to paying patients for compliance with a medical regimen than for improving harmful health-related behaviour: rejection of the former could be based on a feeling of undue influence ('coercion') to accept side effects, however small, whereas rejection of the latter could be based, especially where rewards are involved, on a feeling that bad behaviour should not be indirectly rewarded.

The study was limited in several ways. The scenarios were necessarily different for US and UK participants. UK respondents were asked to make judgements about resource allocation in an existing national health system. To keep the question comparable for the US panel, we asked about a hypothetical 'national health insurance.' US participants show less support to fund any type of treatment than UK participants. This, and some comments made by US participants, reflect an uneasy stance towards national funding of medical treatments. By contrast, the pattern of results relating to financial versus medical interventions, and those relating to rewards versus penalties, are strikingly similar between the two countries. We think this increases validity of our findings regarding acceptability of financial incentives, because it suggests they apply irrespective of such differences in health provision. We do not know how representative our participants are of the UK and US populations, respectively. Members of online panels are convenience samples, but are likely to be more representative of the population in general than other convenience samples commonly used, such as university students.

Our question about fairness was by necessity brief. How different aspects of fairness are perceived in this context is a topic for further research.

\section{Conclusions}

This preliminary study suggests that even if effective, the use of financial incentives to motivate health-enhancing behaviours may be seen to be less acceptable than similarly effective medical interventions. Possible explanations for this finding await replication and extension of the current study.

Funding This study was funded as part of a strategic award from the Wellcome Trust Biomedical Ethics Programme (PI Marteau: 086031/Z/08/Z).

\section{Competing interests None.}

Ethics approval This research was carried out with approval from the King's College London ethics committee (PNM/09/10-89).

Provenance and peer review Not commissioned; externally peer reviewed.

\section{REFERENCES}

1. Lussier JP, Heil SH, Mongeon JA, et al. A meta-analysis of voucher-based reinforcement therapy for substance use disorders. Addiction 2006:101:192-203.

2. Loewenstein G, Brennan T, Volpp KG. Asymmetric paternalism to improve health behaviors. J Am Med Assoc 2007;298:2415-17.

3. Claassen D, Fakhoury WK, Ford R, et al. Money for medication: financial incentives to improve medication adherence in assertive outreach. Psychiatr Bull 2007:31:4-7.

4. Volpp KG, John LK, Troxel AB, et al. Financial incentive-based approaches for weight loss. J Am Med Assoc 2008;300:2631-7. 
5. Cahill K, Perera R. Competitions and incentives for smoking cessation. Cochrane Database Syst Rev 2008;(3):CD004307.

6. Volpp KG, Gurmankin Levy A, Asch DA, et al. A randomized controlled trial of financial incentives for smoking cessation. Cancer Epidemiol Biomarkers Prev 2006:15:12-18.

7. Bauld L, Coleman T. The Effectiveness of Smoking Cessation Interventions During Pregnancy: A briefing Paper. UK Centre for Tobacco Control Studies, Nottingham and Bath, 2009.

8. McColl K. Betting on health. BMJ 2009;338:b1456

9. Marteau TM, Ashcroft RE, Oliver A. Using financial incentives to achieve healthy behaviour. BMJ 2009;338:b1415.

10. Parke H, Brown R, Ashcroft RE, et al. The Public Response to Health Incentives: A Study of UK Media Coverage. Health Expectations (In press).

11. Babcock L, Gelfand M, Small D, et al. Gender differences in the propensity to initiate negotiations. In: Cremer DD, Zeelenberg M, Murnighan JK, eds. Social Psychology and Economics. London: Lawrence Erlbaum, 2006:239-62.

12. R Development Core Team. R: A Language and Environment for Statistical Computing. Vienna, Austria: R Foundation for Statistical Computing. 2009
13. Baayen RH, Davidson DJ, Bates DM. Mixed-effects modeling with crossed random effects for subjects and items. J Mem Lang 2008;59:390-412.

14. Fiske AP, Tetlock PE. Taboo trade-offs: reactions to transactions that transgress the spheres of justice. Polit Psychol 1997;18:255-97.

15. Anderson E. Value in Ethics and Economics. Cambridge, MA: Harvard University Press, 1993.

16. Boyle J. The Public Domain: Enclosing the Commons of the Mind. New Haven \& London: Yale University Press, 2008.

17. Krawiec KD. Show me the money: making markets in forbidden exchange Law Contemp Probl 2009; 72:i-xiv.

18. Rozin P. The process of moralization. Psychol Sci 1999;10:218-21.

19. Rozin P, Singh L. The moralization of cigarette smoking in the United States. $J$ Consum Psychol 1999;8:321-37.

20. Nord E, Richardson J, Street A, et al. Who cares about cost-does economicanalysis impose or reflect social values. Health Policy 1995:34:79-94.

21. Long JA, Helweg-Larsen M, Volpp KG. Patient opinions regarding 'pay for performance for patients'. J Gen Intern Med 2008:23:1647-52.

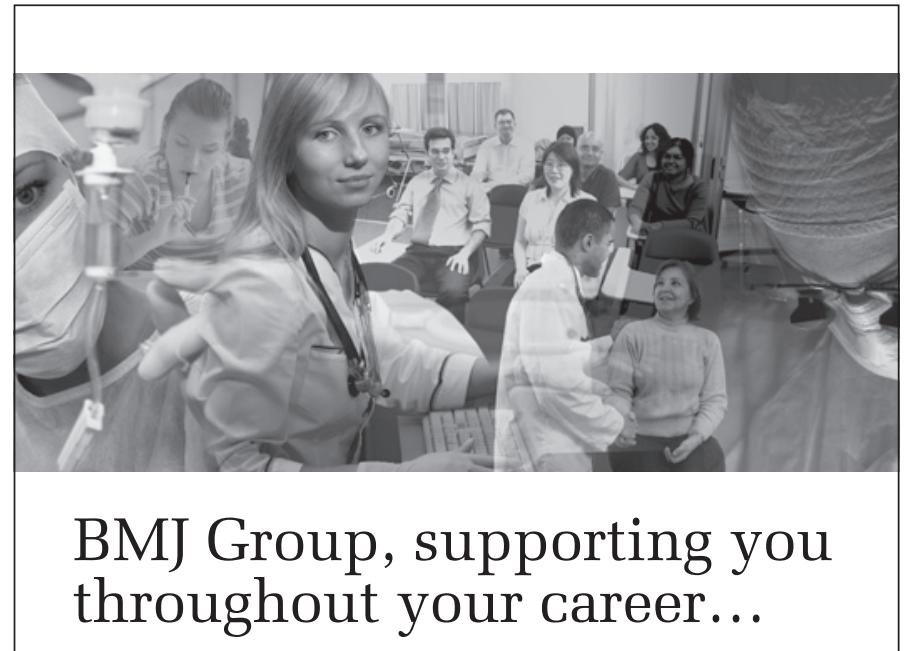

At BMJ Group we have resources available to you at every stage of your career.

Whether you are a medical student or doctor in training looking to keep up with the latest news and prepare for exams, or a qualified doctor who wants the latest medical information, to attend conferences, or looking for your next job, BMJ Group has something to offer. For the latest information on all of our products and services register to receive email updates at

\section{group.bmj.com/registration}

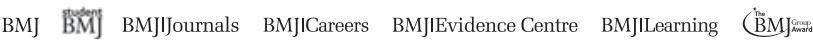

